\title{
Multiaxial Deformations of End-linked Poly(dimethylsiloxane) Networks 5. Revisit to Mooney-Rivlin Approach to Strain Energy Density Function
}

\author{
Takanobu KaWAMURA*, Kenji URAYAmA**, and Shinzo KOHJIYA** \\ ${ }^{*}$ Faculty of Materials and Design, Kyoto Institute of Technology, Matsugasaki, Sakyo-ku, Kyoto 606-8585, Japan \\ ${ }^{* *}$ Department of Material Chemistry, Kyoto University, Sakyo-ku, Kyoto 606-8501, Japan \\ ${ }^{* * *}$ Institute for Chemical Research, Kyoto University, Uji, Kyoto 611-0011, Japan
}

(Received : June 2, 2003)

\begin{abstract}
We strictly assess the validity of the familiar Mooney-Rivlin approach to the strain energy density function $(W)$ on the basis of the quasi-equilibrium uniaxial and biaxial deformation data of end-linked poly(dimethylsiloxane) networks prepared from the solutions with various precursor concentrations. The constants $C_{1}$ and $C_{2}$ in the Mooney equation ( $W$ $\left.=C_{1}\left(I_{1}-3\right)+C_{2}\left(I_{2}-3\right)\right)$ are obtained from the intercept and slope of the linear correlation in the Mooney-Rivlin plots for the uniaxial data, respectively. The biaxial stress-strain relations estimated by the Mooney equation are far from the real behavior for all samples. It is clearly demonstrated that the Mooney-Rivlin approach, which has still been employed due to the simplicity in many studies, is unsuccessful to deduce the form of $W$.
\end{abstract}

Key Words: Rubber elasticity / Elastomer / Biaxial elongation / Mooney-Rivlin equation / Poly(dimethylsiloxane)

\section{INTRODUCTION}

One of the ultimate goals in the physics of rubber elasticity is the determination of the strain energy density function $(W)$ of elastomer. The $W$ corresponds to the elastic free energy stored in a unit volume of elastomer during deformation. The function $W$ for isotropic and incompressible elastic materials is customarily written as a polynominal function of $\left(I_{1}-3\right)$ and $\left(I_{2}-3\right),{ }^{1)}$

$$
W=\sum_{i, j} C_{i j}\left(I_{1}-3\right)\left(I_{2}-3\right)^{j} \text { with } C_{00}=0
$$

where $I_{1}$ and $I_{2}$ are the first and second invariants of Green's deformation tensor and related with the principal ratios along the three orthogonal axes $\left(\lambda_{1}, \lambda_{2}\right.$ and $\left.\lambda_{3}\right)$ as follows.

$$
\begin{aligned}
& I_{1}=\lambda_{1}{ }^{2}+\lambda_{2}{ }^{2}+\lambda_{3}{ }^{2} \\
& I_{2}=\lambda_{1}{ }^{2} \lambda_{2}{ }^{2}+\lambda_{2}{ }^{2} \lambda_{3}{ }^{2}+\lambda_{3}{ }^{2} \lambda_{1}{ }^{2}
\end{aligned}
$$

When the form of $W$ is known, one can calculate the principal engineering stresses $\sigma_{\mathrm{i}}(i=1,2)$ as a function of $\lambda_{\mathrm{i}}(i=1,2)$ using the following relations. ${ }^{2)}$

$$
\sigma_{1}=\frac{\partial W}{\partial \lambda_{1}}=\frac{2}{\lambda_{1}}\left(\lambda_{1}^{2}-\frac{1}{\lambda_{1}^{2} \lambda_{2}^{2}}\right)\left(\frac{\partial W}{\partial I_{1}}+\lambda_{2}^{2} \frac{\partial W}{\partial I_{2}}\right)
$$

** To whom correspondence should be addressed.

E-mail: urayama@scl.kyoto-u.ac.jp

$$
\sigma_{2}=\frac{\partial W}{\partial \lambda_{2}}=\frac{2}{\lambda_{2}}\left(\lambda_{2}^{2}-\frac{1}{\lambda_{1}{ }^{2} \lambda_{2}{ }^{2}}\right)\left(\frac{\partial W}{\partial I_{1}}+\lambda_{1}{ }^{2} \frac{\partial W}{\partial I_{2}}\right)
$$

where the relation $\lambda_{1} \lambda_{2} \lambda_{3}=1$ for incompressibility is used. The direction 3 is the stress-free direction.

The form of $W$ is usually unknown, and some phenomenological functions have been proposed. Among them, the following Mooney equation has been employed as $W$ in many studies.

$$
W=C_{1}\left(I_{1}-3\right)+C_{2}\left(I_{2}-3\right)
$$

For uniaxial deformation $\left(\lambda_{1}=\lambda, \lambda_{2}=\lambda_{3}=\lambda^{-1 / 2}\right)$, the following equation is derived from Eqs.(3) and (4) .

$$
\sigma=2\left(\lambda-\frac{1}{\lambda^{2}}\right)\left(C_{1}+\frac{C_{2}}{\lambda}\right)
$$

Equation (5) indicates that if the plot of $\sigma_{\mathrm{M}}=\sigma /\left(\lambda-\lambda^{-2}\right)$ against $\lambda^{-1}$ (so-called Mooney-Rivlin plot) gives a linear relation, one can obtain $C_{1}$ and $C_{2}$ from the intercept and slope, respectively. The Mooney-Rivlin (M-R) plot often yields a linear correlation for uniaxial data of many elastomers. On the basis of this "apparent" linearity in the M-R plot, the validity of the Mooney equation has often been believed, and there exists many studies utilizing the M-R plot. The M-R approach relying on only simple uniaxial deformation must be the most convenient way to determine the form of $W$, if it is correct. 
However, as demonstrated in the present paper, the linearity in the M-R plot is only apparent, and the resulting $W$ is far from the real form. The qualitatively similar cautions about the M-R approach were made earlier by several researchers: ${ }^{3-7)}$ The Mooney equation determined from the M-R plot could not describe the biaxial elongation data of some bulk-crosslinked rubbers. ${ }^{3-7)}$ Nevertheless, the M-R plot is still often employed in many studies, and there exists many attempts to give a molecular interpretation to the Mooney equation. ${ }^{3), 8)}$ In the present paper, we strictly test again the validity of the M-R approach using the uniaxial and biaxial data of bulk- and solution-crosslinked poly(dimethylsiloxane) networks. We clearly demonstrate the inapplicability of the familiar M-R approach to the evaluation of $W$ irrespective of network concentration at preparation.

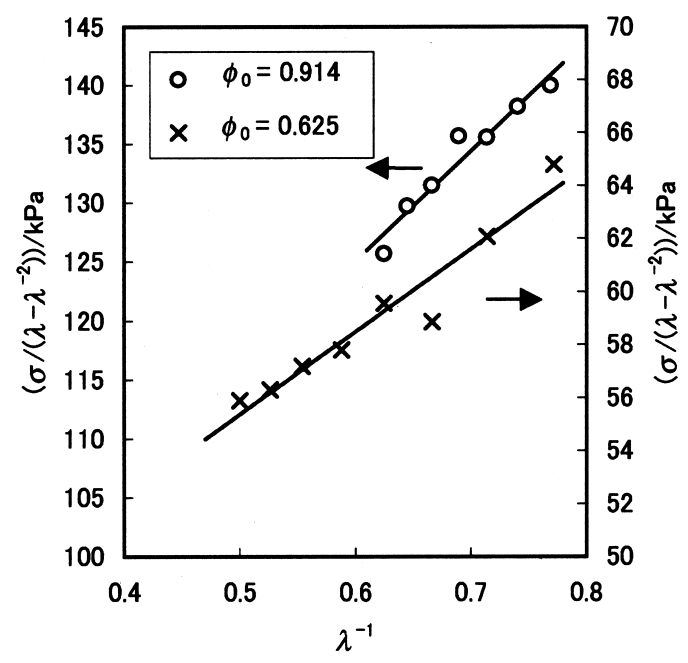

(a)

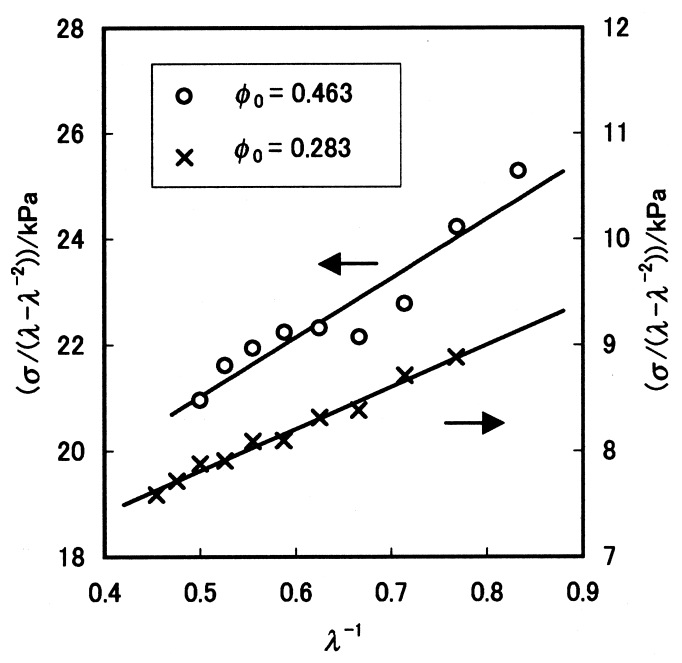

(b)

Fig.1 The Mooney-Rivlin plots of the samples with (a) $\phi_{0}=0.914$ (o) and 0.625 (x) (b) $\phi_{0}=0.463(\mathrm{o})$ and $0.283(\mathrm{x})$

\section{RESULTS AND DISCUSSION}

The details of the samples and measurements are described elsewhere. ${ }^{7), 9)}$ The PDMS networks with different entanglement densities were prepared by end-linking end-reactive bifunctional precursor PDMS with tetra-functional cross-linker in the solutions with different solvent contents. The weight fractions of the precursor PDMS were 1.00, 0.700, 0.500 and 0.300 . The quasi-equilibrium uniaxial and biaxial data were obtained at $40^{\circ} \mathrm{C}$. The data employed here were originally reported in Refs.7 and 9.

Figure 1 shows the Mooney-Rivlin plots for the uniaxial

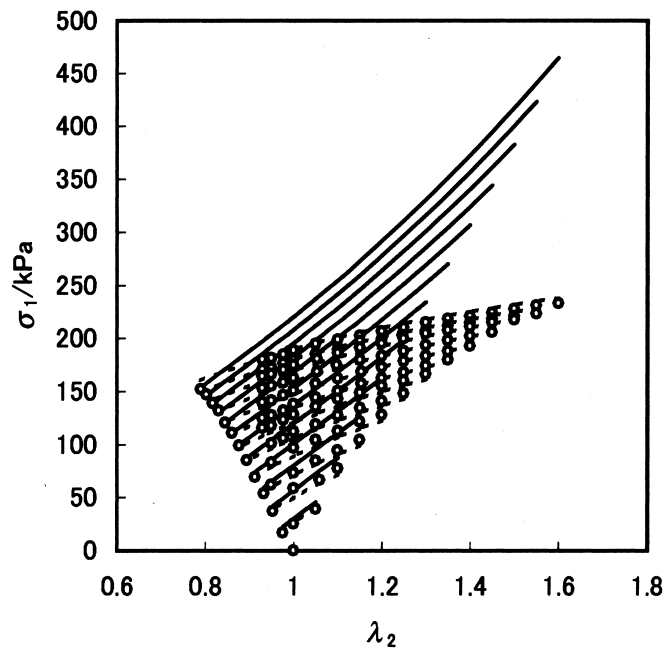

(a)

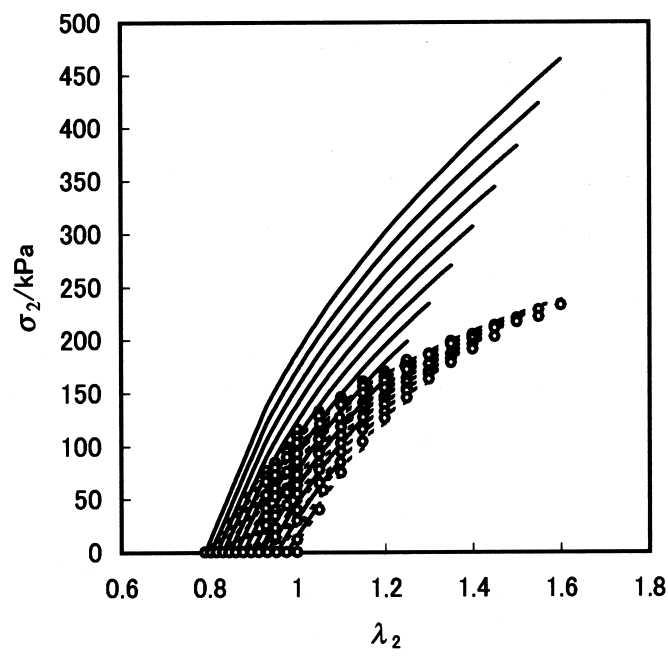

(b)

Fig.2 The $\lambda_{2}$ dependence of (a) $\sigma_{1}$ and (b) $\sigma_{2}$ for the sample with $\phi_{0}=$ 0.914 . The circular symbols are the experimental uniaxial and biaxial data. The solid lines represent the stresses at a constant $\lambda_{1}$ predicted by the Mooney-Rivlin equation with the values of $C_{1}$ and $C_{2}$ obtained by the Mooney-Rivlin plots. The dotted lines depict the stresses at a constant $\lambda_{1}$ predicted by Eq.(7). 
Table I The first and the second constants of Mooney-Rivlin equation ( $C_{1}$ and $C_{2}$, respectively), $C_{2} / C_{1}$, and the root-mean square of errors (rme).

\begin{tabular}{ccccc}
\hline$\phi_{0}$ & $C_{1} / \mathrm{kPa}$ & $C_{2} / \mathrm{kPa}$ & $C_{2} / C_{1}$ & $r m e$ \\
\hline 0.914 & 34.3 & 46.9 & 1.37 & 0.498 \\
0.625 & 19.9 & 15.6 & 0.784 & 0.566 \\
0.463 & 7.71 & 5.60 & 0.726 & 0.583 \\
0.283 & 2.91 & 1.98 & 0.682 & 0.879 \\
\hline
\end{tabular}

elongation data of the samples with $\phi_{0}=0.914,0.625,0.463$, 0.283 . The quantity $\phi_{0}$ is the network volume fraction where the fraction of unreacted materials is subtracted from the preparation concentration. As can be seen in the figure, the data for each sample fall on the straight line, which accords with the general trend of the linear behavior observed in many earlier studies. According to the M-R approach, the intercept or slope of the line is the double of $C_{1}$ or $C_{2}$ in Eq.(4), respectively. The estimated values of $C_{1}$ and $C_{2}$ are listed in Table I. Both $C_{1}$ and $C_{2}$ decrease with a decrease in $\phi_{0}$. The detailed discussion for the $\phi_{0}$ depedence of the constants will be presented later.

One can calculate the uniaxial and biaxial stress-strain relations using Eqs.(3) and (4) with the $C_{1}$ and $C_{2}$ values obtained from the M-R plots. Figures 2-5 illustrate the comparison of the experimental uniaxial and biaxial data with the predictions of the Mooney equation for the samples with $\phi_{0}$ $=0.914,0.625,0.463$, and 0.283 , respectively. Parts a and $\mathrm{b}$ of the figures show the $\lambda_{2}$ dependence of $\sigma_{1}$ and $\sigma_{2}$, respectively, where the direction 2 is the smaller stretching direction in biaxial elongation. The circles and solid lines depict the experimental data and the prediction of Eq.(4) at a constant $\lambda_{1}$. For all samples, evidently, the prediction of Mooney equation overestimates the biaxial stresses over the whole deformation region, and it only agrees with the uniaxial data which was originally used to determine the $C_{1}$ and $C_{2}$ values. Especially, the up-turn behavior of stresses at high biaxial deformations predicted by the Mooney equation is far from the real behavior. These tendencies for the deviation are essentially the same for all samples. We evaluated the root mean square of errors for stresses (rme) to estimate the deviations of the prediction from the experimental data.

$$
r m e=\frac{1}{2 n}\left\{\sum_{i=1,2 m=1} \sum_{m}^{n}\left(\frac{\sigma_{i, \exp }^{m}-\sigma_{i, t h}^{m}}{\sigma_{i, \exp }^{m}}\right)^{2}\right\}^{1 / 2}
$$

where $\sigma_{\mathrm{i}, \mathrm{th}}$ and $\sigma_{\mathrm{i} \text { exp }}$ are the calculated and experimental stresses in the $i$ th direction, respectively, and $n$ is the number of the experimental data point. The value of rme for each sample is listed in Table I and plotted as a function of $\phi_{0}$ in Fig.6. It can be seen that the degree of the deviation is large at any $\phi_{0}$ and slightly increases with decreasing in $\phi_{0}$. These results clearly indicate that the M-R approach is inapplicable to estimate $W$ irrespective of the $\phi_{0}$ value.

The M-R approach fails because (1) the estimation of $W$ relies on the data of uniaxial deformation which is only one particular type of deformation among all possible types of deformation of incompressible materials; (2) the form of the Mooney equation is too simple. The reason (1) is intuitively understandable: The data for many types of deformation are required as a basis to estimate $W$ according to the principle that

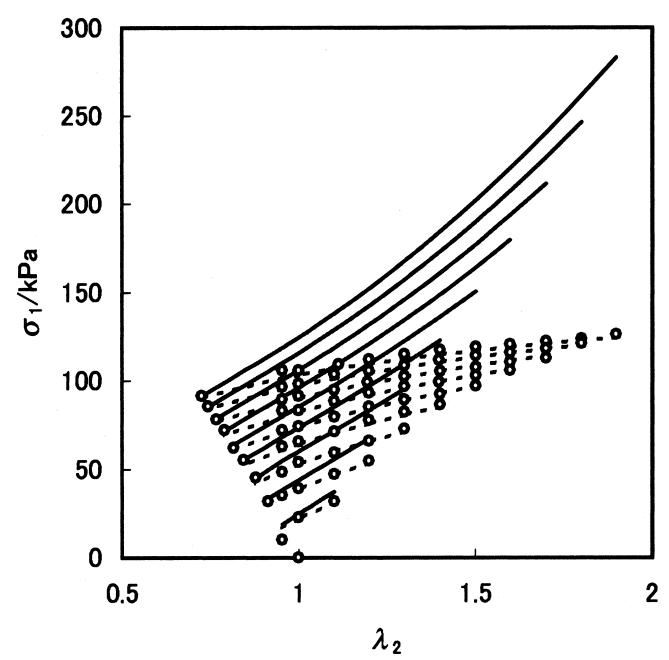

(a)

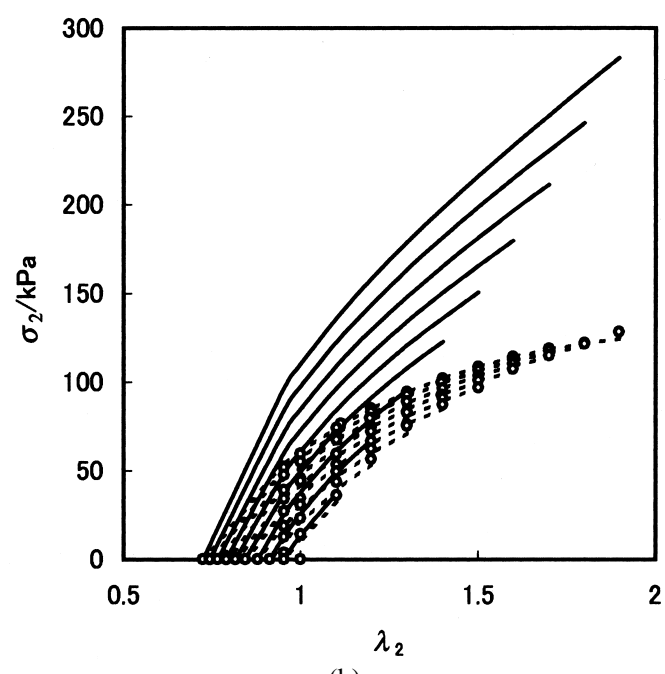

(b)

Fig.3 The $\lambda_{2}$ dependence of (a) $\sigma_{1}$ and (b) $\sigma_{2}$ for the sample with $\phi_{0}=$ 0.625 . The circular symbols are the experimental uniaxial and biaxial data. The solid lines represent the stresses at a constant $\lambda_{1}$ predicted by the Mooney-Rivlin equation with the values of $C_{1}$ and $C_{2}$ obtained by the Mooney-Rivlin plots. The dotted lines depict the stresses at a constant $\lambda_{1}$ predicted by Eq.(7). 
$W$ describes the stress-strain relations under all admissible deformations. The general biaxial deformations where each of the two principal ratios is independently altered cover the whole accessible deformations of incompressible materials. ${ }^{2), 3), 10)}$ In previous studies, we estimate the following form of $W$ for the PDMS networks on the basis of the data of general biaxial deformations. ${ }^{7), 9)}$

$$
\begin{aligned}
& W=\sum_{i, j=0}^{2} C_{i j}\left(I_{1}-3\right)^{i}\left(I_{2}-3\right)^{j} \quad\left(\text { with } C_{00}=C_{12}=C_{21}=C_{22}=0\right) \\
& =C_{10}\left(I_{1}-3\right)+C_{01}\left(I_{2}-3\right)+C_{11}\left(I_{1}-3\right)\left(I_{2}-3\right)+C_{20}\left(I_{1}-3\right)^{2}+C_{02}\left(I_{2}-3\right)^{2}
\end{aligned}
$$

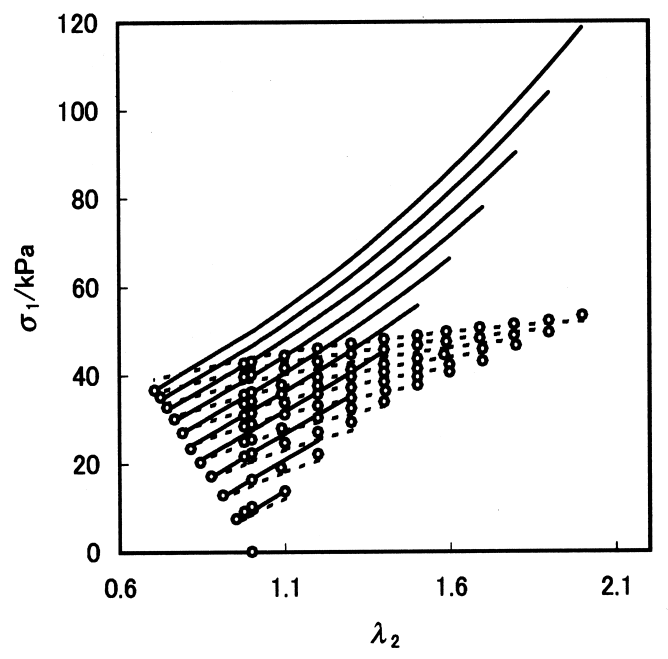

(a)

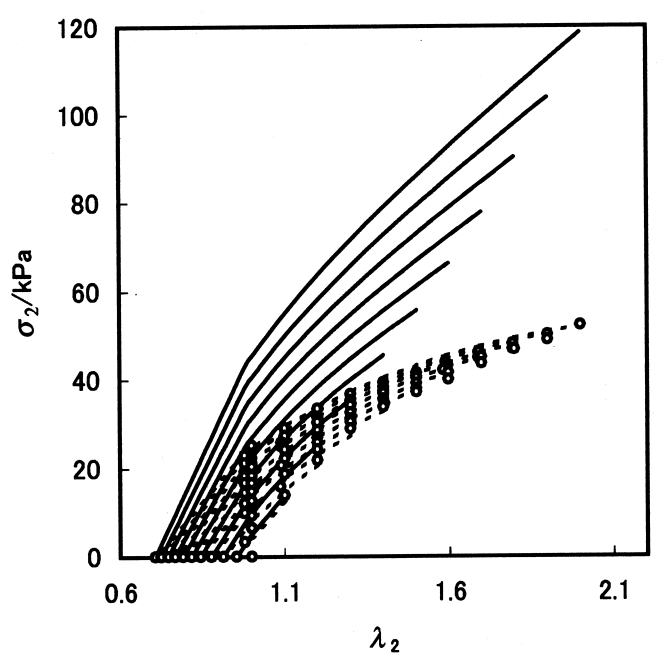

(b)

Fig.4 The $\lambda_{2}$ dependence of (a) $\sigma_{1}$ and (b) $\sigma_{2}$ for the sample with $\phi_{0}=$ 0.463 . The circular symbols are the experimental uniaxial and biaxial data. The solid lines represent the stresses at a constant $\lambda_{1}$ predicted by the Mooney-Rivlin equation with the values of $C_{1}$ and $C_{2}$ obtained by the Mooney-Rivlin plots. The dotted lines depict the stresses at a constant $\lambda_{1}$ predicted by Eq.(7). where $C_{\mathrm{ij}}(i, j=0,1,2)$ depends on $\phi_{0}$. Equation (7) corresponds to the expansion of Eq.(1) up to the second order but with $C_{12}$ $=C_{21}=C_{22}=0$. The stresses at a constant $\lambda_{1}$ calculated by Eq.(7) are also presented in Figs.2-5 by the dotted lines. Equation (7) successfully reproduces the experimental data over the whole deformation region examined, which indicates that Eq.(7) is close to a real form of $W$. As compared to the Mooney function (Eq.(4)), Eq.(7) contains the three extra terms, which well explains the reason (2) for the failure of the M-R approach.

The quantity $C_{2} / C_{1}$ has often been employed as a basis for the molecular interpretation of the elastic behavior of elastomer. ${ }^{3), 8)}$ The first term of the Mooney equation (Eq.(4))

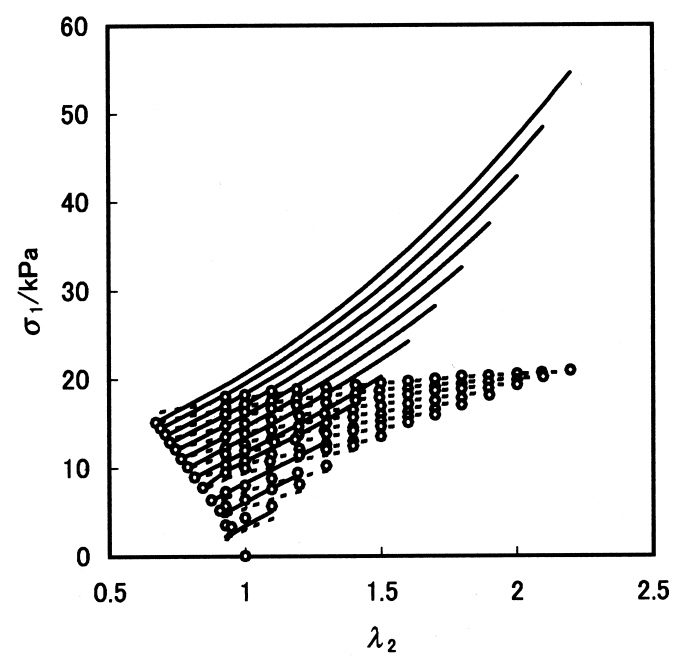

(a)

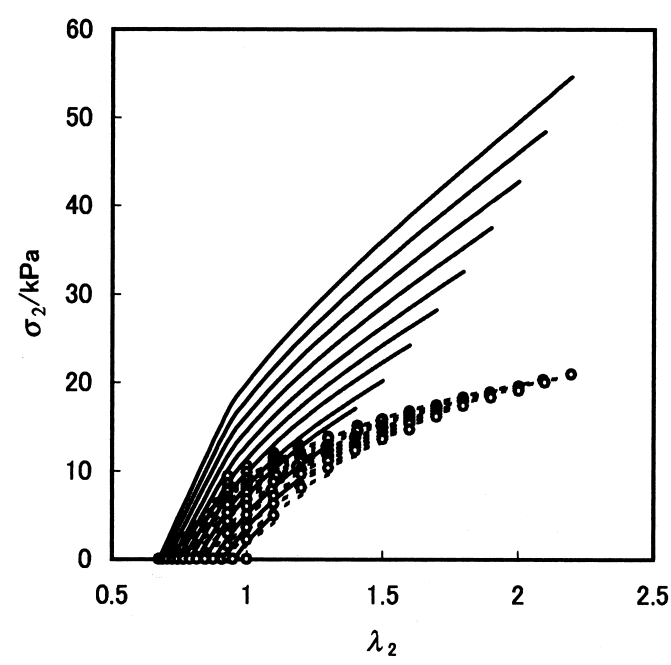

(b)

Fig.5 The $\lambda_{2}$ dependence of (a) $\sigma_{1}$ and (b) $\sigma_{2}$ for the sample with $\phi_{0}=$ 0.283 . The circular symbols are the experimental uniaxial and biaxial data. The solid lines represent the stresses at a constant $\lambda_{1}$ predicted by the Mooney-Rivlin equation with the values of $C_{1}$ and $C_{2}$ obtained by the Mooney-Rivlin plots. The dotted lines depict the stresses at a constant $\lambda_{1}$ predicted by Eq.(7). 
and Eq.(7) corresponds to the elastic free energy of the classical theory of rubber elasticity: $W=C_{1}\left(I_{1}-3\right)$. The ratio $C_{2} / C_{1}$ has often been considered as a measure of the deviation of the real elastic behavior from the classical theory. Figure 6 displays the $\phi_{0}$ dependence of $C_{1}, C_{2}$, and $C_{2} / C_{1}$. Both $C_{1}$ and $C_{2}$ decrease with decreasing in $\phi_{0}$ as a result of dilution effect. The ratio $C_{2} / C_{1}$ largely decreases in the region $\phi_{0}>0.6$, but it is almost constant in the region $\phi_{0}<0.6$. In the previous paper, ${ }^{9)}$ the dependence of the ratio $C_{\mathrm{ij}} / C_{10}(i, j=0,1,2)$ in Eq.(7) on $\phi_{0}$ was investigated. All of $C_{\mathrm{ij}} / C_{10}(i, j=0,1,2)$ steeply decrease with a decrease in $\phi_{0}$ in the region $\phi_{0}<0.6$, which is drastically different from the trend of $C_{2} / C_{1}$ in the M-R approach. The dilution reduces the number of trapped entanglements which is

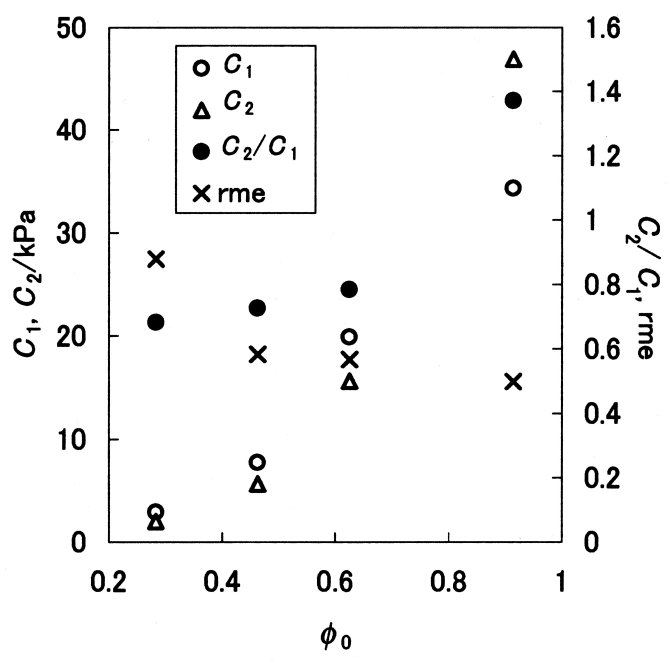

Fig.6 The $\phi_{0}$ dependence of $C_{1}(\mathrm{o}), C_{2}(\Delta), C_{2} / C_{1}(\bullet)$, and root-mean square of errors (rme) $(\mathrm{x})$

not considered in the classical theory, with the result that the elastic behavior of the system approaches to the prediction of the classical theory. Thus it is not admissible that one assigns $C_{2} / C_{1}$ in the M-R approach as a measure of the difference between the real behavior and the prediction by the classical theory. This also indicates that any molecular interpretation utilizing the M-R approach has no definite physical basis. The $\mathrm{M}-\mathrm{R}$ approach provides us only the qualitative information that the observed elastic behavior disagrees with the prediction of classical theory when the $C_{2}$ value is finite. The data of general biaxial elongation achieving all types of admissible deformation are also needed to discuss correctly the elastic behavior of elastomer from the molecular viewpoint. ${ }^{11), 12)}$

\section{REFERENCES}

1) Mooney M, J Appl Phys, 11, 582 (1940).

2) Rivlin RS, Saunders DW, Philos Trans R Soc, A243, 251 (1951).

3) Treloar LRG, "The Physics of Rubber Elasticity", 3rd ed, (1975), Oxford University Press, Oxford, England.

4) Kawabata S, Kawai H, Adv Polym Sci, 24, 89 (1977).

5) Gottlieb M, Gaylord RJ, Macromolecules, 20, 130 (1987).

6) Fukahori Y, Seki W, Polymer, 33, 502 (1992).

7) Kawamura T, Urayama K, Kohjiya S, Macromolecules, 34, 8252 (2001).

8) Erman B, Mark JE, "Structures and Properties of Rubberlike Networks", (1997), Oxford University Press, Oxford, NY, and references therein.

9) Kawamura T, Urayama K, Kohjiya S, J Polym Sci Part B. Polym Phys, 40, 2780 (2002).

10) Tschoegl NW, Gurer C, Macromolecules, 18, 680 (1985).

11) Urayama K, Kawamura T, Kohjiya S, Macromolecules, 34, 8261 (2001).

12) Urayama K, Kawamura T, Kohjiya S, J Chem Phys, 118, 5658 (2003). 
